\title{
Die schwarze Pädagogik des 21. Jahrhunderts
}

Kinder zu schlagen ist verboten, weder dürfen das Eltern noch Lehrende oder Erziehende. Die Kinderrechtskonvention forderte das Recht auf gewaltfreie Erziehung 1989 ein. Weit weniger im Bewusstsein ist, dass gewaltfreie Erziehung auch entwürdigende Maßnahmen und seelische Verletzungen miteinschließt.

Tatsächlich belegen Zahlen, dass die körperliche Gewaltanwendung sowohl in den Familien als auch in den Institutionen deutlich abgenommen hat. Ein Klaps auf den Hintern oder eine Ohrfeige wird „nur“ noch von ca. $10 \%$ aller Eltern in Deutschland als Erziehungsmittel eingesetzt. In Frankreich und in der Schweiz ist man da weniger zimperlich. Erst vor ein paar Monaten wurde das Schlagen von Kindern in Frankreich gesetzlich verboten, Schweizer Kinder darf man immer noch ungestraft ohrfeigen. Nach einer Studie der Zürcher Hochschule für Angewandte Wissenschaften (ZHAW) und der Haute Ecole de Travail Social (HETS) Fribourg vom Juli 2018 haben rund zwei Drittel aller Jugendlichen elterliche Gewalt in der Erziehung erlebt. Allerdings gibt es auch dort eine große Initiative, die das Recht auf Unversehrtheit gesetzlich verankern will.

Alles in Allem bewegt sich etwas. Das Bewusstsein erwacht dafür, dass die Integrität der Kinder nicht durch Schläge verletzt werden darf.

Nur: Wenn andere, subtilere Erziehungsmaßnahmen, die seelische Verletzungen verursachen, eingesetzt werden, sind wir damit wirklich einen Schritt weiter in unseren Erziehungsmethoden? Diese werden oft nicht mal als seelische Gewalt gewertet, beispielsweise, wenn ein Kind nach einem Streit ignoriert, angeschwiegen oder isoliert wird. Nach wie vor ist es üblich, ein Kind auf sein Zimmer zu schicken, wenn es nicht gehorcht, auch schon Zweijährige werden auf diese Weise bestraft. Ausgrenzung aus der Gemeinschaft verletzt das Kind und bedeutet gleichzeitig Liebesentzug. (,Geh auf dein Zimmer! Dich will ich jetzt erst mal nicht mehr sehen, bis du zu dich wieder benimmst."). 